ISSN 1794-9831

E-ISSN 2322-7028

Vol. 12 No. 1

Ene - Jun 2015

Cúcuta, Colombia

20 de Marzo

de 2015

* Psicóloga.

Especialista en

Epidemiología.

Docente de la

Universidad

Nacional de

Rosario. Correo

electrónico:

stella.m.orzuza@

gmail.com

** Descriptores

en Ciencias de la

Salud (DeCS), en

la página $\mathrm{http}: / /$

decs.bvs.br/E/

homepagee.htm

de la Biblioteca

virtual en salud del

proyecto BIREME,

de la Organización

Mundial de la

Salud y de la

Organización

Panamericana de

Salud.

\title{
Brechas de equidad: análisis de la información sociodemográfica de la población indígena †
}

\author{
Stella Maris Orzuza*
}

\section{RESUMEN}

Objetivo: caracterizar socio-demográficamente a la población indígena residente en la provincia de Santa Fe de Argentina. Materiales y Métodos: se realizó un análisis estadístico descriptivo de las variables investigadas en la población indígena residente en la provincia de Santa Fe en Argentina, con base en la información recopilada en los Censos Nacionales de Población, Hogares y Viviendas 2001 y 2010 en la República Argentina. La población fue indagada a través de encuestas domiciliarias. Su pertenencia étnica fue determinada por autoadscripción. Se encuestaron 368.893 hogares en el relevamiento censal 2010. Resultados: la proporción de la población indígena en Argentina respecto al conjunto, representa menos del $3 \%$ y se está reduciendo. Por otra parte se estableció que mientras que para el total nacional la población de 65 años o más, representa cerca del $10 \%$, con un punto más elevado en la provincia de estudio, para los pueblos originarios de esta provincia la cifra no supera el 7\%. Según el último relevamiento censal el hacinamiento se ha reducido menos aceleradamente en la población indígena que en la población general de la provincia aumentando la brecha entre ambas poblaciones. En cuanto a la calidad de las viviendas donde habita la población indígena se observa que un $29,5 \%$ son deficitarias, superando en 12 puntos a la media nacional. Conclusiones: El análisis de la información sociodemográfica de la población indígena deja en evidencia la postergación y situación de desventaja de esta población en relación a los totales nacionales y provinciales para la población general.

PALABRAS CLAVES: demografía, determinantes sociales de la salud, población indígena, política de salud.*

Para citar este artículo / To reference this article / Para citar este artigo

Orzuza SM. Brechas de equidad: análisis de la información sociodemográfica de la población indígena. Rev. cienc. cuidad. 2015; 12(1): 14-26.

\footnotetext{
† El presente artículo presenta resultados parciales de la investigación titulada "Estudio epidemiológico sobre la situación de salud de la infancia étnica residente en la ciudad de Rosario (provincia de Santa Fe, Argentina) en el año 2012”, bajo la dirección de la Mg. Ana Cecilia Augsburger, realizado con el apoyo del programa de becas "Ramón Carrillo-Arturo Oñativia", categoría individual otorgada por el Ministerio de Salud de la Nación en el año 2012, a través de la Comisión Nacional Salud Investiga.
} 


\section{Equity gaps: analysis of socio-demographic information concerning to indigenous population}

\section{ABSTRACT}

Objective: to characterize socio-demographically to the indigenous population living in the province of Santa $\mathrm{Fe}$ in Argentina. Materials and Methods: it was conducted a descriptive statistical analysis of the inquired variables in the indigenous population who live in the province of Santa Fe in Argentina by the National Census of Population and Housing 2001 and 2010 in Argentina, comparatively with the general population. The population was inquired through household surveys and determined the belonging ethnicity by auto registration. 368.893 households were inquired in the census survey 2010 . Results: The magnitude of the indigenous population in Argentina represents less than $3 \%$ of the population and it is decreasing day by day. While the national total population, older than 65 years represent about $10 \%$, with a higher point in the province of this study, for the indigenous people from this province the number does not exceed $7 \%$. According to the latest census survey overcrowding has fallen less rapidly among the indigenous population than in the general population of the province increasing the gap between the two populations. In relation with the quality of housing where the indigenous population lives. It shows that $29.5 \%$ of houses are deficient for the whole country, surpassing by 12 points the national average of substandard housing. Conclusions: The analysis of the socio-demographic information about the indigenous population shows the postponement and disadvantage of this population in relation to national and provincial totals for the general population.

KEYWORDS: demographics, social determinants of health, indigenous population, health policy. 
ISSN 1794-9831

E-ISSN 2322-7028 Vol. 12 No. 1

Ene - Jun 2015

Cúcuta, Colombia

\section{Lacunas equidade: análise de informações sociodemográficas da população indígena}

\section{RESUMO}

Objetivo: caracterizar sócio-demograficamente o indígena população que vive na província de Santa Fe, na Argentina. Materiais e Métodos: a análise estatística descritiva das variáveis indagadas a população indígena que vive na província de Santa $\mathrm{Fe}$, na Argentina pelo Censo Nacional da População e Habitação 2001 e 2010 na Argentina, em comparação com a população em geral foi realizado . A população foi investigado em através de inquéritos aos agregados familiares e determinados por etnia autoadcripción. 368893 famílias no recenseamento perguntou 2010. Resultados: o tamanho da população indígena na Argentina representa menos de 3\% da população está encolhendo. Enquanto a população total nacional de 65 anos e representa cerca de $10 \%$, com um ponto mais alto na província de estudo, para os povos indígenas desta província o número não superior a $7 \%$. Segundo o último levantamento censitário superlotação ele caiu menos rapidamente entre a população indígena que na população em geral da província aumentou o fosso entre as duas populações. Quanto à qualidade da habitação onde a população indígena mostra que $29,5 \%$ das casas são deficientes para todo o país, superando em 12 pontos do que a média nacional de habitações precárias. Conclusões: a análise das informações sociodemográfico sobre a população indígena mostra o adiamento e desvantagem desta população em relação aos totais nacionais e provinciais para a população em geral.

PALAVRAS-CHAVE: demografia, determinantes sociais da saúde, política de saúde da população indígena. 


\section{INTRODUCCIÓN}

$\mathrm{L}$ a población de América Latina se integra por un amplio espectro de herencias y ascendencias culturales que permite identificar algunos grupos poblacionales como étnicos/raciales. Estos grupos, con el mayor tiempo de radicación en el territorio americano, junto a las comunidades afrodescendientes, representan más del $25 \%$ de la población total de la región (1).

En los últimos años, especialmente impulsada desde organismos internacionales, ha crecido la preocupación y visibilización de la situación de salud de los grupos étnicos, en particular, de los pueblos originarios. Se señala críticamente que presentan un menor acceso a los servicios de salud y perfiles salud enfermedad más complejos (2).

Esta situación desfavorable ha sido abordada desde la perspectiva de derechos considerando la mayor vulnerabilidad y menor equidad en salud de estos pueblos, incluyendo su histórico relegamiento, sometimiento, expropiación de sus tierras y migración forzada. Coincidentemente, en la IV Conferencia Interparlamentaria de Salud y Pueblos Indígenas, Afrodescendientes y otras etnias se señaló que es políticamente injustificable desde el punto de vista social y una violación a los derechos humanos persistir con una situación de desigualdad que afecta al 25\% de la población de la región (3).

En el ámbito académico se ha reconocido a la condición étnico-racial como causal de desigualdades e inequidades en el campo de la salud (4). Ha sido ampliamente investigado por la epidemiología social, así como por las ciencias sociales relacionadas con la salud, documentándose en las últimas décadas por organismos internacionales, gobiernos nacionales, movimientos indígenas y organizaciones no gubernamentales que comprenden la situación desfavorable y de rezago que padecen las minorías étnicas respecto a sus condiciones de vida y de salud (5).

No obstante este reconocimiento unánime, se advierte que la información producida sobre la situación de salud de los pueblos indígenas de América Latina es escasa e inespecífica. Haro (6) menciona que diversos autores latinoamericanos enmarcan sus estudios epidemiológicos bajo el nombre de epidemiología sociocultural, señalando la necesidad de ampliar los referentes teóricos y metodológicos en el estudio de la relación entre etnicidad y salud.
La desigual propensión a padecer determinados problemas de salud por características sociales diversas tales como riqueza, educación, ocupación, raza/etnia, género y condiciones de la vivienda ha sido documentada principalmente a partir del siglo XIX. Para explicar la desigual distribución entre la población de los problemas saludenfermedad-atención, se desarrollaron teorías sobre la determinación social de los procesos saludenfermedad derivados del materialismo dialéctico e inscriptos en la epidemiología crítica latinoamericana.

Si bien son diversas las teorías que han buscado comprender las desigualdades sociales en salud, todas coinciden en que las desigualdades en salud están fuertemente entrelazadas con la organización social y tienden a reflejar el grado de inequidad existente en cada sociedad (7-10).

En relación con los grupos étnicos, la consideración de las desigualdades sociales lleva a una doble determinación: la posición social que ese grupo ocupa en la sociedad y la aceptación/rechazo que puedan tener frente a los grupos mayoritarios. El significado concreto que la raza/etnia adopta como variable social predictora de los estados de salud es modelado por el medio histórico, socioeconómico, cultural y epidemiológico, siendo prácticamente imposible establecer reglas universales sobre las relaciones entre raza/etnia y salud. Por ello, los determinantes sociales de la salud necesitan ser comprendidos en contextos particulares (4).

En el plano legislativo, desde fines de los ochenta del siglo pasado se han realizado reformas constitucionales en los países latinoamericanos que reconocen el carácter pluricultural y pluriétnico de los países (11). En torno a esta problemática, Argentina, en el plano legislativo con la reforma de la Constitución Nacional del año 1994, avanzó en la garantía de derechos para esta población, reconociendo su preexistencia étnica y cultural en el territorio nacional, además de garantizar el respeto a su identidad, el derecho a una educación bilingüe e intercultural, la posesión y propiedad comunitaria de las tierras fiscales que tradicionalmente ocupan y regulando la entrega de otras aptas y suficientes para el desarrollo humano.

Por otro lado, en el año 2000 se ratifica el convenio $\mathrm{N}^{\mathrm{o}} 169$ de la Organización Internacional del Trabajo (OIT), uno de los instrumentos jurídicos 
ISSN 1794-9831

E-ISSN 2322-7028

Vol. 12 No. 1

Ene - Jun 2015

Cúcuta, Colombia internacionales más actualizados sobre la materia (12). Sin embargo, a pesar de expresar actualmente una extensa legislación indigenista en el plano constitucional, los pueblos originarios vieron y ven limitado el ejercicio de sus derechos (13). La amplitud de la proclama de derechos y la amplitud de su garantía difieren significativamente.

Esta situación de inequidad se ha perpetuado y consolidado a lo largo del tiempo. Para los pueblos originarios, la constitución del Estado Nación argentino implicó la invisibilización de sus culturas y la dificultad para su transmisión, la negación de sus propias formas de organización política así como la ruptura de amplias territorialidades donde las comunidades convivían y se desplazaban. Así también, desembocó en persecuciones, sometimiento y una fuerte reducción del tamaño de su población (14-15). Todavía es necesario un largo camino para revertir lo que tantos años de sometimiento ha provocado.

Para alcanzar políticas sanitarias inclusivas que permitan superar las profundas brechas de inequidad que afectan a los pueblos originarios es necesario conocer su situación de salud. Según el Foro de Investigación en Salud de Argentina (16), en este país la población indígena presenta un perfil epidemiológico complejo atravesado por una mayor mortalidad y vulnerabilidad en comparación con el resto de la población, se identifican enfermedades infecto-contagiosas evitables como desnutrición infantil, chagas y tuberculosis, así como casos de discriminación y racismo en los servicios hacia los usuarios indígenas.

Sin embargo, una seria limitante de la información epidemiológica sobre esta población es que la misma no se recupera regularmente y la poca información existente no se encuentra sistematizada, lo cual impide la generación de políticas públicas e intervenciones acordes con las necesidades de salud de los pueblos. Si bien la Organización Mundial de la Salud (OMS) insta a la incorporación de la variable étnica en los registros sanitarios desde el año 1993, en Argentina todavía no se ha realizado, impidiendo identificar la situación sanitaria de esta población (17).

Uno de los elementos fundamentales para avanzar en el diseño de políticas públicas adecuadas y pertinentes en función de las necesidades específicas de los diversos grupos de población es la construcción de información sociodemográfica, puesto que ésta permite identificar las brechas de equidad existentes en relación con el resto de la población (18).

Es necesario que esta información sea la base de las políticas públicas para reducir las diferencias y las inequidades, además de promover el desarrollo integral de los pueblos indígenas en el marco de sus principios y su cosmovisión. Para poder garantizar la universalidad de las políticas de desarrollo y protección de derechos y el acceso a ellas de todos y todas, resulta imprescindible conocer, de forma específica, el comportamiento que los indicadores muestran para cada uno de los grupos, diferenciados por su pertenencia o autoadscripción a un grupo étnico/cultural particular (18).

Los últimos relevamientos censales realizados en Argentina (2001 y 2010) han incluido la variable étnica para la identificación de las personas descendientes o pertenecientes a pueblos indígenas, permitiendo identificar la expresión de diversos indicadores de población, vivienda y hogares en esta comunidad en particular. Así también, su inclusión en el censo poblacional 2001 permitió realizar la encuesta complementaria de Pueblos Originarios 20042005, a partir de la cual se identificó que el 23,5\% de sus hogares encuentran sus necesidades básicas insatisfechas; una proporción muy alta comparada con la media nacional de $14,3 \%$.

La tasa de analfabetismo para la población indígena de 10 años o más es de $9.1 \%$, tres veces mayor frente a la tasa nacional, que es de $2.6 \%$. La comparación de los resultados de los censos 2001 y 2010 permite identificar que no solamente la población indígena es una minoría (representa menos del $3 \%$ de la población, según el censo nacional 2010) sino que, además, se está reduciendo. Mientras la población indígena decrece, en la población general se observa un crecimiento del $0,9 \%$ anual.

Los pueblos originarios presentan valores desfavorables en la mayoría de los indicadores sociodemográficos y una mortalidad y morbilidad más elevada. Esta información permite establecer una caracterización general de la situación desigual de los pueblos indígenas. Por otro lado, la posibilidad de su desagregación en unidades geográficas menores, avanza en la identificación de situaciones particulares que pueden presentarse en cada provincia, información necesaria para la definición de programas y políticas particulares. 


\section{MATERIALES Y MÉTODOS}

Para caracterizar sociodemográficamente a la población originaria residente en la provincia de Santa Fe se realizó un análisis estadístico descriptivo de las variables indagadas en la población indígena por los Censos Nacionales de Población, Hogares y Viviendas 2001 y 2010, comparativamente con la población general. Para el análisis comparativo se recurrió al Censo Nacional de Población, Hogares y Viviendas 2001 y 2010 realizado por el Instituto Nacional de Estadísticas y Censos (19-20). Se recuperó la información desagregada correspondiente a la población indígena para la provincia de Santa $\mathrm{Fe}$ y para la población general.

Para la obtención de la información correspondiente al Censo 2001 se utilizó la base de datos Redatam y se consultaron las tablas elaboradas y publicadas por el organismo para la información de dicho censo. La pertenencia étnica fue establecida en ambos relevamientos a través de la autoadcripción. Este criterio se ha adoptado internacionalmente y es la metodología utilizada en Argentina para identificar a la población indígena. La autoadcripción o autoreconocimiento se realiza mediante una pregunta que consulta si se reconoce como perteneciente o descendiente de un pueblo indígena.

Los indicadores analizados fueron: población, población por sexo y grandes grupos, hogares, hacinamiento, calidad de las viviendas, viviendas deficitarias, propiedad de la vivienda y el terreno, principal combustible utilizado para cocinar, agua de red. Se realizó un análisis estadístico descriptivo comparativo. Es necesario aclarar que los indicadores analizados en el presente artículo son todos los que han sido indagados en ambos relevamientos censales para la población originaria.

La información relevada en el año 2001 correspondió a 64.176 habitantes, mientras que al relevamiento censal 2010 respondieron 48.265 personas pertenecientes o descendientes de pueblos originarios. La información fue resumida en tablas y gráficos realizados con el programa informático Microsoft Office Excel versión 2007.

Este estudio fue sometido al Comité de Ética del Instituto de la Salud Juan Lazarte y al Comité de Ética de Atención Primaria de la Salud de la Municipalidad de Rosario, Santa Fe, Argentina, siendo evaluado favorablemente en ambas ocasiones.

\section{OBJETIVOS}

\section{Objetivo general}

Analizar la situación de salud de la población perteneciente o descendiente de la etnia qom ${ }^{\dagger \dagger}$ en una localidad de la provincia de Santa Fe, Argentina, en el año 2012.

\section{Objetivos específicos $^{\dagger \dagger \dagger}$}

- Caracterizar socio-demográficamente, según condiciones de vida, a la población étnica

- Describir las particularidades culturales de la etnia qom relacionadas con los procesos salud-enfermedad.

- Identificar los problemas de salud/salud mental registrados en el centro de salud municipal para la población seleccionada.

- Indagar la percepción de los adultos sobre los problemas de salud de las niñas y los niños qom y las estrategias de resolución del problema.

\section{RESULTADOS Y DISCUSION}

\section{Caracterización socio-demográfica}

\section{Población: lento crecimiento de la población indígena}

La población perteneciente o descendiente de pueblos originarios, residente en la provincia de Santa $\mathrm{Fe}$, se redujo durante el periodo 2001 a 2010 en un $24,7 \%$, pasando de 64.176 a 48.265 habitantes; esta tendencia se observa también a nivel nacional, aunque de forma menos acentuada; mientras que la población indígena a nivel nacional representaba en el año 2001 el 3\%, para el año 2010 esta cifra se redujo a 2,3\%. En

Cúcuta, Colombia

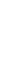

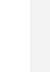


ISSN 1794-9831

E-ISSN 2322-7028

Vol. 12 No. 1

Ene - Jun 2015

Cúcuta, Colombia contraste, la variación intercensal relativa para la población general del país ha sido de $10,6 \%$ de signo positivo, tendencia reflejada en todas las provincias, con valores que oscilan entre el $4 \%$ y el $39 \%$.

Si se compara la proporción de población indígena argentina con otros países de Latinoamérica, se observa que la situación es heterogénea. Mientras que en Bolivia más del $60 \%$ de su población se identifica como indígena, otros países presentan dimensiones poblacionales menores al $2 \%$ como es el caso de Brasil, Costa Rica y Paraguay (23).

Con respecto a los hogares en términos absolutos, la cantidad de hogares con al menos una persona descendiente de pueblos originarios, arrojada por el Censo 2010, es de 368.893 correspondiente al 3,03\% del total de hogares de Argentina. El análisis comparativo con el censo 2001 sobre el crecimiento poblacional indica que sólo 10 de las 24 provincias argentinas presentan un crecimiento de signo positivo en el volumen de hogares respondientes de la variable étnica; esto indica que la población indígena ha crecido de forma menos acelerada que el resto de la población. Las provincias que presentan un crecimiento mayor al 1\% son Chubut, Neuquén, La Pampa y Río Negro.

En la provincia de Santa Fe, los hogares con descendientes de pueblos originarios constituyen el $1,8 \%$ del total provincial, representando a 18.938 hogares según el censo poblacional 2010 (19). Con relación a la distribución por sexo, en el año 2001 las mujeres representaron el $50,4 \%$ de la población indígena de la provincia, apenas sobrepasando por un $0,4 \%$ a los varones con el $49,6 \%$, mientras que esta situación se invierte en el censo 2010, representando los varones el $51,3 \%$ de la población indígena de la provincia, 2,6\% más que las mujeres, que fue de $48,7 \%$.

La estructura de la población con respecto a los grupos quinquenales no refleja grandes variaciones en los diez años transcurridos. Se observa el envejecimiento de la población a través de una reducida base de la pirámide poblacional, tendencia conservada desde el relevamiento censal anterior, al igual que la pirámide poblacional correspondiente a la población general de la provincia.

El grupo más numeroso se encontró entre las edades 15-64 años, equivalente al 60,6\% de la población en el año 2001 y el 65,4\% en el año 2010. En segundo lugar se encuentra el grupo más joven de 0-14 años, siendo en el año 2001 de 32,9\% y para el año 2010 el 29\%. Finalmente, el grupo de 65 años y más representó en el año 2001 al $6,5 \%$ de la población, mientras que para el censo 2010 representaba el 5,7\%, como puede observarse en la figura 1.

Figura 1. Pirámide poblacional, pueblos originarios residentes en la Provincia de Santa Fe, Argentina.

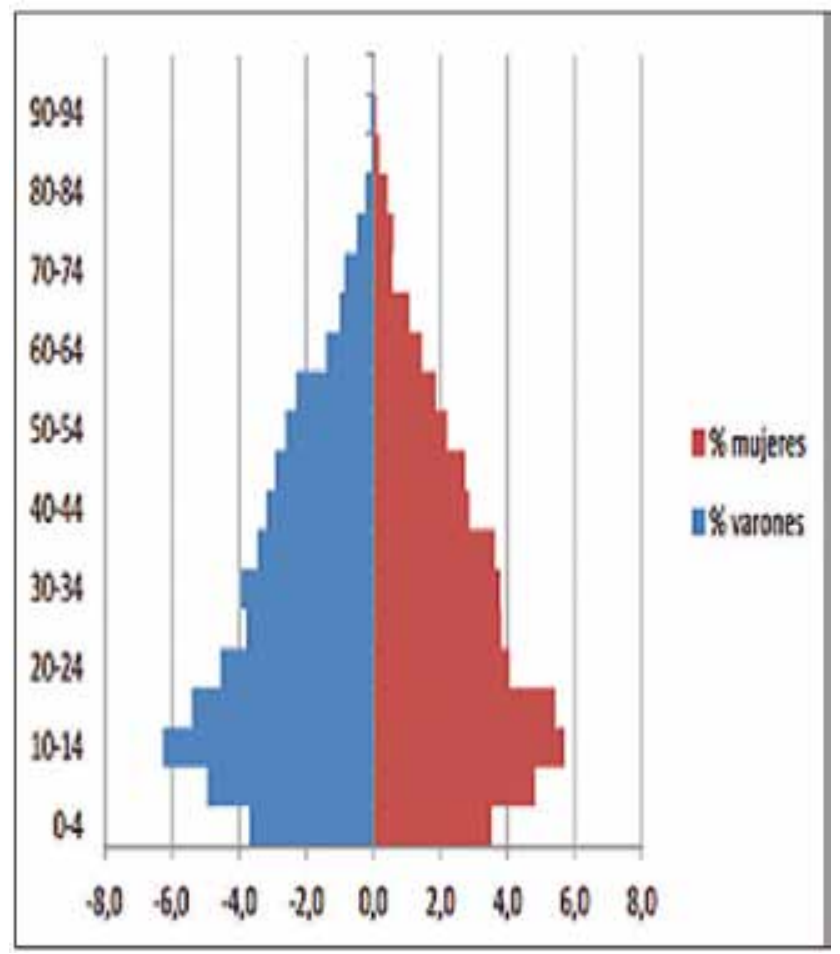

Fuente: Censo Nacional de Población, Hogares y Viviendas. Indec, 2010.

$\mathrm{Al}$ atender al grupo de 65 años y más puede observarse que la población correspondiente a esta franja etaria perteneciente a pueblos indígenas fue sustancialmente menor que la presente en el conjunto de la población argentina. Mientras que para el total nacional la población de 65 años y más representó cerca del $10 \%$, siendo un punto más elevado en la provincia de Santa $\mathrm{Fe}$, para los pueblos originarios de esta provincia la cifra no superó el 7\%. Esta situación podría estar denotando una mortalidad más elevada entre los pueblos aborígenes, producto de una deficiente situación social y, más específicamente, de una precaria situación de salud (24).

La participación elevada de los grupos en edades activas puede deberse a la migración interna. El estudio de Del Popolo y Oyarce (25), señala que la migración interna es selectiva por edad (emigran 
relativamente más las personas en edades jóvenes), configurando áreas indígenas muy envejecidas pero a la vez con alta presencia de niñas y niños, fenómeno que se da principalmente en zonas rurales.

En el caso de la provincia de Santa Fe, la importante presencia de jóvenes adultos se ha justificado, de acuerdo con Fernández y Stival (26), por el carácter migratorio de parte de la población perteneciente a pueblos originarios que reside muchas veces sólo temporalmente en esta provincia. La mayor densidad poblacional de las ciudades de Santa Fe -con mayor oferta de empleos y mejores condiciones de vida que las provincias y localidades de origen de la población migrante- favorece el proceso migratorio, asentándose, en general, en los cordones de pobreza que rodean las ciudades.

El movimiento migratorio de la población indígena desde sus tierras de radicación originaria hacia la periferia de grandes centros urbanos es un fenómeno que se observa en la mayoría de los países latinoamericanos. Sin embargo, el panorama regional es heterogéneo, puesto que existen países donde la población indígena conserva el predominio rural, como sucede en Panamá (25).

Labaja fecundidad, observada en la reducida población 0-4 años, coincide con los resultados obtenidos en la población general; en ambos relevamientos poblacionales, 2001 y 2010, la provincia de Santa Fe presentó una estructura etaria que refleja el efecto de baja fecundidad (27).

Según el estudio realizado por Del Popolo y Oyarce (23) sobre el perfil sociodemográfico de la población indígena y afrodescendiente en Latinoamérica, existe una alta fecundidad y mortalidad para esta población, con estructuras etarias más jóvenes respecto a la población no indígena. La provincia de Santa Fe, por su parte, se diferencia del análisis realizado por estos autores mostrando una baja fecundidad.

Acerca de las pirámides poblacionales comparadas entre población indígena y no indígena en diversos países, algunas comparten la similitud entre la estructura por edad y sexo entre población indígena y no indígena, como sucede en la provincia de Santa $\mathrm{Fe}$, mientras otras expresan claramente la desigualdad en estos indicadores, como ocurre en Panamá y Costa Rica (23).

Es importante aclarar que, siendo la autoadcripción el principal criterio utilizado en la región para la identificación de esta población, son necesarias algunas consideraciones al respecto. Una primera es que los resultados pueden estar afectados por la mala declaración de la edad, que suele darse en los censos de la región y sobre todo en las personas de edad avanzada, motivada por falaces registros de nacimientos, el analfabetismo e incluso por una concepción distinta de la temporalidad (28).

Un segundo aspecto se refiere a que como consecuencia de los procesos de aculturación y negación de la identidad, se presenta una mayor auto-identificación en los adultos mayores que en las cohortes más jóvenes. Sin embargo, se debe señalar que los procesos de revitalización cultural también se dan proporcionalmente más entre los jóvenes, sólo que aparentemente no alcanzan a compensar los efectos del fenómeno anterior (25).

\section{Viviendas: mayor expresión de la desigualdad social}

En el relevamiento censal del año 2001 para la población general de la provincia de Santa Fe, el hacinamiento representaba el 7,9\% mientras que en la población indígena se identificaron 1.461 viviendas, es decir, el 9\% con presencia de hacinamiento crítico. En el relevamiento censal 2010 este indicador muestra un crecimiento de signo positivo para la población general de la provincia de Santa Fe, alcanzando 3,2\% de las viviendas. No ocurre lo mismo con la población indígena, donde el descenso del hacinamiento es mucho menos acelerado, representando el 7,2\%.

En cuanto a la calidad de las viviendas en donde habita la población indígena, según la cuarta publicación del censo 2010 se observa que un $29,5 \%$ de sus viviendas son deficitarias para el total del país; esto supera en 12 puntos a la media nacional de viviendas deficitarias. En cambio, en la provincia de Santa Fe, en el año 2001 el 40,7\% de sus viviendas fueron consideradas deficitarias, mientras que en el año 2010 este indicador descendió tres puntos. Para la población total de la provincia, este indicador presenta una variación intercensal de 5 puntos representando un $17,3 \%$ de las viviendas en el año 2001 y un $12,4 \%$ en el año 2010. Se presenta su representación gráfica para facilitar la comprensión a través de la figura 2 . 
ISSN 1794-9831
Figura 2. Vivienda deficitaria, comparación pueblos originarios y población general de la Provincia de Santa Fe, Argentina.

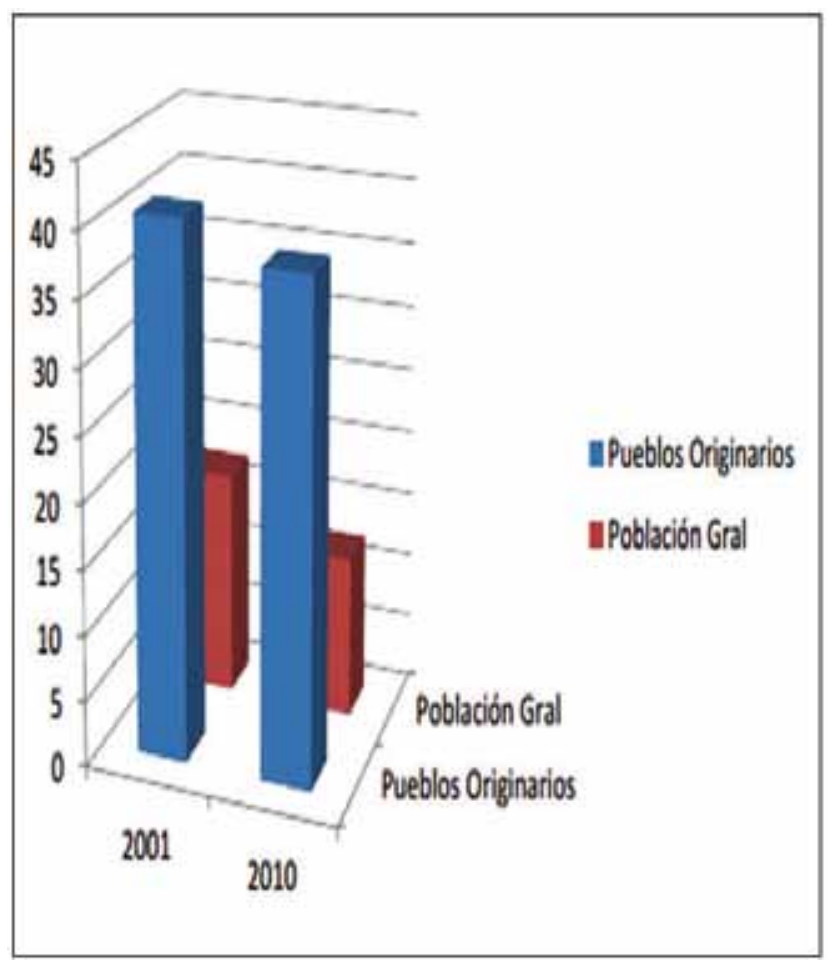

Fuente: Censo Nacional de Población, Hogares y Viviendas. Indec, 2010.

En cuanto a la propiedad de la vivienda y el terreno, a nivel nacional el $63,3 \%$ de los hogares indígenas ha declarado ser propietario tanto de la vivienda como del terreno. Las cifras son similares para la provincia de Santa Fe, donde el 61,8\% de los hogares declaró ser propietario de la vivienda y el terreno. Sin embargo, esto no significa que el $63,3 \%$, que representa 233.656 hogares, haya solucionado el reclamo histórico respecto de la propiedad de la tierra. El censo no contempla la cosmovisión indígena sobre este tema, no indaga si esos terrenos son de tipo comunitario o si se ajustan a las tierras reclamadas históricamente por los pueblos originarios.

El principal combustible utilizado para cocinar es el gas para el $85,1 \%$ de los hogares indígenas del país; casi el 50\% dispone de gas de red y algo más del 35\% utiliza gas envasado. Comparando estos datos con la media nacional, se observa que los hogares con descendientes de indígenas se encuentran 6,4 puntos porcentuales por debajo de la media nacional que utiliza gas de red y 2 puntos respecto de los que usan gas envasado.
Por último, es llamativo el porcentaje de uso como combustible de leña o carbón, ubicado 8,9 puntos por encima de la media nacional. Esta categoría se abulta particularmente en aquellas provincias donde el gas de red no es el servicio más utilizado, como es el caso de Catamarca, Formosa, Jujuy, Chaco, Misiones y Santiago del Estero.

En la provincia de Santa Fe según el Censo 2010, el $97,5 \%$ de las viviendas utilizan como principal combustible para cocinar gas en red, en tubo o en garrafa. Comparativamente con el relevamiento censal 2001, se observa que la utilización de gas como principal combustible para cocinar ha aumentado en 3 puntos para esta población, mientras que, en particular, la utilización de gas de red, que es la forma de suministro de gas más económica en esta provincia, aumentó 5 puntos.

Sobre la utilización de agua de red, a nivel nacional se observa que hay una brecha de 4,4 puntos desfavorable para los hogares indígenas en comparación con la media nacional según el relevamiento censal 2010. Es importante observar que en la categoría agua de lluvia, río, canal, arroyo o acequia, los hogares analizados se encuentran 2,5 puntos por encima de la media nacional.

En la provincia de Santa $\mathrm{Fe}$, según el mismo relevamiento censal, la provisión de agua a las viviendas se realiza principalmente a través de la red pública, llegando a 15.519 hogares, adoptando un valor porcentual de $84,4 \%$, levemente superior al porcentaje nacional de $81.9 \%$.

A nivel provincial, el $69 \%$ de las viviendas que se abastecen con agua corriente la reciben en el interior de la vivienda. El abastecimiento por agua de lluvia, arroyo, canal o río se reduce a 54 viviendas. Comparativamente con el relevamiento censal 2001, este indicador refleja una mayor accesibilidad a un recurso tan imprescindible para esta población como lo es el agua. También se evidencia que ha aumentado en 13 puntos el acceso a agua corriente de la red pública y aumentó en tres puntos el acceso a agua corriente en el interior de la vivienda. Por otro lado, se redujeron considerablemente las viviendas que se abastecen principalmente por agua de lluvia, arroyo, canal o río.

En lo concerniente a indicadores de condiciones de vida, es necesario señalar que el primer relevamiento consultado para este estudio, realizado en el año 
2001, se efectuó en el marco de un contexto de crisis económica. Sólo presentaron un crecimiento de signo positivo los indicadores sociales y económicos luego del año 2003.

La variación intercensal favorable en la mayoría de los indicadores seleccionados, tanto en población general como en población indígena entre los censos 2001 y 2010, encuentra su explicación en la situación económica, social y política de los periodos en que ambos censos fueron realizados. Sin embargo, ello no explica la situación de desventaja de los pueblos originarios, que muestra un crecimiento menos acelerado en sus indicadores comparativamente con la población general.

En los años 2001 y 2002 se vivió una profunda crisis económica, política y social en Argentina que marcó el fin de la convertibilidad y la caída del gobierno nacional. Esta crisis interrumpió un ciclo de la historia argentina reciente, iniciado tras la crisis hiperinflacionaria de 1989, que estuvo signado por la hegemonía del paradigma neoliberal, el cual impregnó el discurso y la práctica de la mayor parte de la clase política, las corporaciones empresarias y sindicales y los medios de comunicación hegemónicos (29).

La población argentina, en general, vio reducida su capacidad adquisitiva, el desempleo aumentó y la clase trabajadora vio incrementada la precariedad en sus condiciones de trabajo. A partir de entonces comenzó un ciclo que requirió de las nuevas autoridades nacionales la realización de importantes cambios en la forma de hacer política nacional (30). situación se vio reflejada en los indicadores sociodemográficos.

Mientras que la población argentina con necesidades básicas insatisfechas (NBI) representaba en el censo 2001 el 17,7\%, para el relevamiento 2010 se redujo a $12,5 \%$. La misma variación intercensal se observó en el mismo indicador para la provincia de Santa $\mathrm{Fe}$ con un $14,8 \%$ en el año 2001 y $9,5 \%$ en el año 2010 $(19-20)$.

En cuanto al hacinamiento, en 2001 se registró un $4,8 \%$ en hogares y $8,8 \%$ de población hacinada, mientras que en 2010 estos valores disminuyen a $4 \%$ en hogares y 7,3\% en población. Para el año 2001 se

\footnotetext{
\$ Las casas tipo B son las viviendas que cumplen al menos una de las siguientes condiciones: tienen piso de tierra, no tienen provisión de agua por cañería dentro de la vivienda o no disponen de baño con descarga de agua (19).

registró que del total de hogares de todo el país, el $15,6 \%$ habitaban en casas tipo B ; para el 2010 este porcentaje se redujo al 13\% (19). El hacinamiento es alto para el relevamiento 2001 pero se encontró marcadamente reducido en el censo 2010 para el total de la población.

Sin embargo, en relación con la población originaria, la disminución del hacinamiento es menos acelerada, denotando una situación de desventaja de los pueblos originarios en comparación con la población general. Mientras que la diferencia en el censo 2001 era de dos puntos entre la población general y la población indígena, esta diferencia se acentúa en el relevamiento 2010, aumentando la distancia entre ambos grupos poblacionales.

Lo mismo se observa en el indicador de vivienda deficitaria, mientras que para la población general de la provincia de Santa Fe desciende cinco puntos en el censo 2010, para los pueblos originarios no alcanza a descender 3 puntos. Pero en este indicador la brecha entre la población indígena y la población general es mucho más marcada en términos porcentuales, mientras que representa el $17,3 \%$ para la población general en el año 2001, el 40,7\% de las viviendas en las que residen las personas descendientes o pertenecientes a pueblos originarios, es decir, más de un tercio de esta población son deficitarias. Esta diferencia se mantiene en el relevamiento poblacional 2010.

La utilización de gas para cocinar y el agua de red, si bien muestran un incremento de signo positivo intercensal, reflejan también la postergación de esta población en relación a la población general de la provincia, donde estos indicadores crecen de manera más acelerada.

Si bien no pudieron recuperarse otros indicadores de condiciones de vida, los datos arrojados por la Encuesta Complementaria de Pueblos Originarios 2004-2005 (31), el Fondo de las Naciones Unidas para la Infancia (Unicef) (32), Cuyul (33) y Domancich y Rodríguez (33) dan cuenta de la situación de pobreza en que vive la mayoría de la población indígena en Argentina y su limitado acceso a servicios públicos.

\section{CONCLUSIONES}

El análisis de la información sociodemográfica de la población indígena muestra la postergación y situación de desventaja de esta población. Tomando 
ISSN 1794-9831

E-ISSN 2322-7028

Vol. 12 No. 1

Ene - Jun 2015

Cúcuta, Colombia sólo algunos indicadores que han podido recuperarse, tanto a nivel nacional como para la provincia de Santa $\mathrm{Fe}$, con el fin de comparar las informaciones obtenidas, se evidencia el sincretismo de situaciones en los distintos niveles que, con pequeñas variaciones, evidencian la postergación de una población por siglos invisibilizada.

Como señala Rojas, según Del Popolo y Oyarce (23), no es posible comprender la situación sociodemográfica actual de los pueblos indígenas y las brechas de acceso a los bienes y servicios del Estado que estas poblaciones presentan en comparación con la población no indígena en América Latina, sin remitirse a la historia de la conquista y colonización, caracterizada por la devastación y el genocidio.

De acuerdo con Del Popolo y Oyarce (25), Albó señala críticamente que se estima que, en los primeros 100 años de conquista, la población originaria se redujo en un $75 \%$ por guerras, por la llamada invasión mórbida y por la dominación a sangre y fuego de las culturas indígenas como respuesta a la necesidad de expandir el dominio de la Corona Española y Portuguesa en el continente, proceso que se basó en el convencimiento de que los pueblos originarios, su visión de mundo y su naturaleza eran inferiores.

Sostenidos en estas afirmaciones, Del Popolo y Oyarce (23), asumen que los determinantes históricos en esta región del continente americano dejaron una sociedad cuyas relaciones de poder se basaron en la legitimación del dominio de la cultura occidental sobre la indígena, amazónica y afrocaribeña, dependiendo del área geográfica, país o grupo étnico. Estas prácticas discriminatorias, presentes desde el momento del contacto, sirvieron -y sirven hoy todavía- para definir y perpetuar dicha dominación, de acuerdo con determinantes culturales y económicos que se refuerzan mutuamente.

La accesibilidad a servicios públicos es un indicador idóneo para analizar el cumplimiento del derecho a las prestaciones que el Estado se ha comprometido a garantizar para la totalidad de la población. Los grupos sociales que no tienen acceso a ellos ven negados sus derechos sociales a servicios públicos básicos. Este reducido análisis demuestra que para la población originaria de Argentina se mantiene la reproducción de la histórica postergación que ha padecido pese a los avances jurídicos y normativos en materia de derechos.
La brecha observada en los indicadores sociodemográficos pone de manifiesto la importancia de realizar estudios similares sobre indicadores sanitarios y educativos. En la actualidad, estos estudios acerca de la situación socio-sanitaria de estos pueblos no pueden realizarse debido a que los registros sanitarios no permiten la identificación de esta población en la provincia de Santa Fe.

La inclusión de la pregunta de identificación étnica en el Censo 2001 es la primera iniciativa estadística fundamentada en el reconocimiento constitucional de los pueblos indígenas y en la ratificación del convenio 169 de la Organización Internacional del Trabajo (OIT) sobre pueblos indígenas y tribales. Es un importante avance, ya que las desigualdades sociales en los pueblos indígenas sólo pueden ser contempladas en la toma de decisiones, si existe información estadística que permita su identificación (35).

Se considera importante aclarar, finalmente, que en este artículo no fue posible incluir información correspondiente a la localidad de la provincia de Santa $\mathrm{Fe}$, lugar donde se realizó el estudio del cual se desprende este texto, debido a que los datos correspondientes todavía no han sido publicados, a pesar del tiempo transcurrido y de las estimaciones realizadas por el organismo responsable del relevamiento, que pronosticaban su difusión oficial en diciembre de 2011. Esta población continúa estando subordinada a prácticas segregacionistas y arbitrarias que impiden la posibilidad de establecer políticas públicas que reduzcan las inequidades y permitan el resarcimiento histórico necesario para con estos pueblos. 


\section{REFERENCIAS BIBLIOGRÁFICAS}

1. Organización Mundial de la Salud/Organización Panamericana de la Salud. Grupo étnico y salud. 132 Sesión del Comité Ejecutivo. Washington D.C: OMS; 2003.

2. CEPAL. Salud de la población joven indígena en América Latina. Un panorama general. Santiago de Chile: Naciones Unidas; 2011.

3. Organización Panamericana de la Salud. IV Conferencia Interparlamentaria de Salud y Pueblos Indígenas, Afrodescendientes y otras etnias. Sao Paulo, Brasil: OPS; 2005.

4. Barata R. Cómo y por qué as desigualdades sociais fazem mal á saúde. Rio de Janeiro: Editora Fiocruz; 2009.

5. Haro J. Etnicidad y salud. Estado del arte y referentes del noroeste de México. Región y sociedad. 2008; 20, (2) número especial: 265-312.

6. Haro J. Epidemiología convencional, epidemiología sociocultural y salud colectiva. Requerimientos para un diálogo entre disciplinas. I Congreso Internacional de Transdisciplinariedad, Mexicali, México: Universidad Autónoma de Baja California; 2010 Marzo 16-18.

7. Breilh J. Epidemiología: economía, medicina y política. Santo Domingo, República Dominicana: Secretaría de Estado de Salud Pública y Asistencia Social; 1981.

8. Marmot M, Wilkinson RG. Social determinants of health. London: Oxford Press; 1999.

9. Samaja JA. A reproduçao social e a saúde: elementos teóricos e metodológicos sobre a questao das relaçoes entre saúde e condiçoes de vida. Salvador: Casa da Saúde; 2000.

10. Hofrichter R. Health amd social justice. San Francisco: John Wiley \&Sons; 2003.

11. Del Popolo F, Oyarce AM, Ribotta B. Sistema de indicadores sociodemográfico de poblaciones y pueblos indígenas de América Latina - SISPPI. Santiago: CEPAL/CELADE - Fondo indígena; 2005.

12. Mereminskaya E. El Convenio 169 de la OIT sobre pueblos indígenas y tribales: Derecho internacional y experiencias comparadas. Estudios Públicos. 2011; 121: 213-276.

13. Barié C. Cap. III Breve recuento histórico sobre las legislaciones y los indios. En: Barié C. Pueblos indígenas y derechos constitucionales en América Latina: un panorama. $2^{\circ}$ Ed. Bolivia: Editorial Abya Yala; 2003.

14. Rojas R. Diversidad cultural y étnica en la Región y situación de la salud de los pueblos indígenas de las Américas. Encuentro Regional para el análisis del acceso a tratamiento anti-tuberculoso en poblaciones indígenas: Documento memoria. Panamá: Organización Panamericana de la Salud; 2004 Oct 13-15.

15. Montenegro R, Stephens C. Indigenous health in Latin America and the Caribbean. Lancet. 2006; 367: $1859-69$.

16. Foro de Investigación en Salud de la Argentina (FISA). Estado de conocimiento y agenda de prioridades para la toma de decisiones en situación de salud, intervenciones y líneas de investigación para la toma de decisiones en salud con pueblos indígenas en Argentina. Resumen Ejecutivo. [Internet]. Buenos Aires: Ministerio de Salud de la Nación; 2008. [consultado 21 de enero de 2013]. Disponible en: http://www.saludinvestiga.org.ar/policys comision.asp

17. Cuyul A, Robetto M, Specogna M. Pueblos indígenas y sistemas de información en salud: la variable étnica en seis provincias argentinas. Revista Argentina de Salud Pública. 2011; 2 (7): 12-18.

18. Schoenfeld $P$. Inclusión de la variable étnica en las fuentes de información sociodemográfica del Ecuador. Santiago de Chile: Naciones Unidas; 2009.

19. Instituto Nacional de Estadísticas y Censos (INDEC) Cuarta publicación. Resultados definitivos. Censo Nacional de Población, Hogares y Viviendas 2010. Análisis de datos. [consultado 21 de mayo de 2012]. Disponible en: http://www.indec.gov.ar.

20. Instituto Nacional de Estadística y Censos (INDEC). Censo Nacional de Población, Hogares y Viviendas 2001. Resultados publicados. [consultado 21 de mayo de 2012]. Disponible en: http://www.indec.gov.ar.

21. Orzuza S. Problemas de salud de niñas y niños indígenas: mejorar sus condiciones de vida para garantizar el derecho a la salud. Direito em Debate. 2013; 22 (40): 91-117.

22. Orzuza S. Concepciones y prácticas indígenas sobre la salud y la enfermedad. Conocerlas para respetarlas. Revista Sujeto, Subjetividad y Cultura. 2013; 5 (abr): 67-78.

23. Del Popolo F, Oyarce AM. Población indígena de América Latina: perfil sociodemográfico en el marco de la CIPD y de las Metas del Milenio. Seminario Internacional. Chile: CEPAL; 2005.

24. Pantelides E, Moreno M. Situación de la población en la Argentina. $1^{\circ}$ ed. Buenos Aires: Programa Naciones Unidas para el Desarrollo; 2009.

25. Del Popolo F, Oyarce AM. Pueblos indígenas y afrodescendientes de América Latina y el Caribe: información sociodemográfica para políticas y programas. Santiago de Chile: CEPAL; 2006.

26. Fernández F, Stival M. Políticas, sentidos y vulnerabilidad sociocultural asociados al VIH-Sida en las poblaciones qom de Rosario, Argentina. Desacatos. 2011; 35: 29-40. 
ISSN 1794-9831

E-ISSN 2322-7028

Vol. 12 No. 1

Ene - Jun 2015

Cúcuta, Colombia
27. Instituto Provincial de Estadísticas y Censos (IPEC) Análisis del crecimiento poblacional. Provincia de Santa Fe. Santa Fe: Ministerio de Gobierno y Reforma del Estado: 2008.

28. Del Popolo F. Los problemas en la declaración de la edad de la población adulta mayor en los censos. Serie Población y Desarrollo. Santiago de Chile: CEPAL; 2000.

29. Castellani A, Schorr M. Argentina: convertibilidad, crisis de acumulación y disputas en el interior del bloque de poder económico. Cuadernos del Cendes. 2004; 21 (57): 55-81.

30. Huper P. El estado posnacional: más allá de kirchnerismo y antikirchnerismo. Buenos Aires: Pie de los Hechos; 2011.

31. Instituto Nacional de Estadística y Censos [INDEC] Encuesta Complementaria de Pueblos Indígenas 2004-2005. [consultado 21 de mayo de 2012]. Disponible en: http://www.indec.gov.ar/webcenso/ECPI/index_ecpi.asp.

32. Fondo de las Naciones Unidas para la Infancia. Situación socioeducativa de niñas, niños y adolescentes de Comunidades Toba. Buenos Aires: Unicef; 2011.

33. Cuyul A. La política de salud para pueblos indígenas en el "Impenetrable", provincia de Chaco, Argentina. En: Hirsch S, Lorenzetti M, Salomon O. Procesos de investigación e intervención en salud en comunidades indígenas de la Argentina. Buenos Aires: Ministerio de Salud de la Nación; 2014.

34. Domancich N, Rodríguez Monti C. Análisis Situacional de Salud de Comunidades Toba - Qom. Municipios de Pampa del Indio y General San Martín, Chaco. Informe de Investigación. La Plata: Facultad de Ciencias Médicas, Universidad Nacional de La Plata; 2008.

35. Cuyul A, Rovetto M, Specogna M, Abriata, G. Enfoque étnico en los sistemas de información en salud: la incorporación del enfoque intercultural en los sistemas de información en salud en las provincias de Jujuy, Salta, Formosa, Chaco, Misiones y Neuquén. Reporte investigación. [Internet]. Buenos Aires: Secretaría de Extensión Universitaria y Bienestar Estudiantil. Facultad de Medicina. UBA; 2010 [consultado 17 de marzo de 2012]. Disponible en: http://www.saludindigena.org.ar

\section{BIBLIOGRAFIA}

Instituto Nacional de Estadística y Censos - INDEC. Censo Nacional de Población, Hogares y Viviendas 2010 Censo del Bicentenario. Resultados definitivos, Serie B, No 2. $1^{\circ}$ ed., Buenos Aires: INDEC; 2012. 